\title{
The frame turns also: Factors in differential rotation in pictures
}

\author{
THOMAS O. HALLORAN \\ University of Maryland, Asian Division, Seoul, Korea
}

\begin{abstract}
When pictures of simple shapes (square, diamond) were seen frontally and obliquely, (1) the shapes with a deeper extent into pictured space underwent more rotation (Goldstein, 1979), which is an apparent turning to keep an orientation toward an observer's changing position; (2) there was little effect of whether the observer knew the picture surface's orientation in real space, except that such knowledge could prevent multistability; and (3) depicted picture frames also rotated. In other experiments, figural and frame rotations were independent of each other, and rotation was shown for real frames. The rotation of depthless depictions suggests that at least two rotational factors exist, one that involves the object's virtual depth and one that does not. The nature of this second factor is discussed. Frame rotation appeared to subtract from object rotation when the two were being compared; this could explain a paradox in picture perception: Depicted orientations often seem little changed over viewpoints, despite (apparent) rotations with respect to real-space coordinates.
\end{abstract}

Pictures exhibit very different visual properties than real objects do, when an observer moves around them. Yet the picture's resemblance to the object is usually about as satisfactory from one viewpoint as from another, within a relatively large range of viewing positions (Cutting, 1987; Pirenne, 1970), and the paradox is not well understood.

Some recent research has centered on object rotation. This can be described in terms of constant aspect (Goldstein, 1979; Gombrich, 1978): From wherever we look at a picture, we see the same side, the same portion, of a depicted object; we cannot see its other side by moving around it. This, as we move around, makes pictured things appear to rotate toward us. Portraits follow us with their eyes, as their real subjects would have to do for the same visual effect. But some depictions rotate more than others, for unclear reasons. Any pictured thing that points directly toward us will continue to do so at any viewpoint. Things pointing slantwise or off to the side, though, do not rotate as strongly; some seem to more or less keep their orientation relative to the wall on which the picture is hanging, rather than relative to us passing by. Goldstein demonstrated this variable turning, and called it differential rotation.

(The word rotation, whenever applied herein to the depiction or its surface or frame, refers to a phenomenal turning or repositioning, not to an actual one or to any image manipulation.)

Cutting (1988), Goldstein $(1979,1987)$, and Halloran (1989) have shown that the amount of rotation probably

The author wishes to thank Robert Fox and another reviewer of a previous version of this article. He also thanks all his unpaid subjects. For reprints, please write to T. O. Halloran, University of Maryland, Asian Division, Unit 5060, Box 0100, APO AP 96328-0100. depends on information within the depictive array itself. But these authors have also suggested, each in a distinct way, that some relation between the picture and its surface or frame may be involved in the final perception. At least two kinds of this relation are possible: Either (1) knowledge of the picture surface directly influences a depicted object's perceived direction from the observer, or (2) the influence is indirect, in that knowing the surface's location does not determine the object's apparent direction in the observer's space, but does help decide what orientation has originally been depicted. Evidence against (1) and for (2) will be given.

If rotation does occur regardless of surface knowledge, there must be particular information in the picture itself to cause the entire rotation, and this information is differential over pictures. Differential information exists in the depicted object's depth into virtual space (the apparent space behind the picture), in proportion to its virtual width. This proportion seems plausibly simple, relative to perceptual processes its use might imply, and previous data suggest that objects that either have a proportionately greater virtual depth, or more strongly direct attention to virtual depth, rotate more (Goldstein, 1979, 1987; Halloran, 1989). The idea resembles an older one. Gombrich (1978, p. 158) says that full rotation arises from " a sense of depth combined with an unforeshortened portion of an object that appears to lie quite close to the frontal plane."

Objects that are practically depthless also rotate a little (Goldstein, 1979). This suggests that, if depth causes rotations, some other factor also can, at least sometimes.

The picture's boundary or frame might also rotate, just as Thouless's (1931) geometric figures did. If so, this could tend to preserve, over viewpoints, the depicted relation between object and picture-plane orientations. This in turn might produce "compensation for viewpoint," the 
apparent ability to interpret pictures as if they were being seen frontally (Kubovy, 1986).

The concerns, then, are whether the perceptual source of object rotation is or is not wholly within the object's depiction per se; whether there might be both depthdependent and depth-independent factors in this rotation (and what they might be); whether the frame rotates tooand why; and what that might have to do with a resultant perception of object orientation.

\section{EXPERIMENT 1}

This experiment explored the relation between frame and picture, and the properties of each, as these might affect object differential rotation.

To the extent that depth or constant aspect might cause rotation, it does not depend on the picture surface. And evidence suggests that the observer may not need to locate the picture's surface to see its rotation (Cutting, 1988; Halloran, 1989). It follows that rotation should persist when relevant picture-surface cues are entirely absent. Such cues were removed, first by making redepictions that, seen frontally, appeared as the originals would when seen from aside, and then by removing the (roughly trapezoidal) projection of the original border/frame from the redepiction.

In additional as-if-aside redepictions, the frames' trapezoidal depictions remained present. The apparent orientation of these "frames" was specifically observed. A frame should undergo Thouless's (1931) "regression to the real," by which he meant a consistent tendency for such shapes, seen aslant, to appear as if they were more nearly frontal to their observer than they really are. This is rotation, and if frames rotate, that should affect the picture perceptions to which the surface orientation is relevant, since the frame can indicate surface orientation (Halloran, 1989).

If depicted depth, versus depicted width, determined rotation, it could happen in this way: Viewed from the station point (in correct perspectives, this is the picturetaking/making viewpoint), an object's width is across the surface of the picture; depth is orthogonal to that. When the line of sight is aslant to the picture, however, these dimensions are no longer orthogonal. Width is still tied to the surface direction. For example, lines across an object's width, which were depicted as more or less horizontally parallel, will now be less so; the object visibly tapers across its width-and so does the frame, whether that matters-all toward the same vanishing point. This situation specifies geometrically (whether perceptually) a common direction which is at a slant, not an orthogonal, to the observer. For depth, however, the reverse is true. Depth recedes from the observer, at any viewpoint, rather than from the picture, partly because its gradients (tapers) are vertical, and hardly change their vanishing point when seen from aside. (Views from above or below the picture are not considered here.) Because the specifiers of depth and width so easily become nonorthogonal, rotation could depend on which has the greater depicted extent. A deep extent into virtual space could call atention to distance specifically from the observer's momentary viewpoint; the object would rotate a lot. To the contrary, a wide object, with its plenteous clues that the object was not being depicted in a plane normal to viewpoint, ought to rotate less. (The general fact that the geometries of nonstandard virtual space can be nonrectilinear is discussed in Cutting [1987, 1988] and in Halloran [1989].)

In sum, this experiment tested two values of depth/width ratio of a depicted object, and the presence versus the absence of framing, for their effects on object rotation. In addition, judgments were made of the perceived orientation of the frame under simulated sidewise viewing. As possible confounds, the effects of object-edge orientation, and the presence or absence of a perspective background for the stimulus figures, which might link those figures more firmly to the frame, were examined.

\section{Method}

Subjects. The 7 subjects were 3 United States Air Force commissioned officers ( 2 of whom were aircrew members), 1 noncommissioned officer, a manufacturing supervisor, a retired teacher, and the experimenter. The 4 military personnel and the supervisor were students in a University of Southern California extramural graduate program in systems management. Ages ranged from 24 to 67 years; 2 subjects were female. All subjects reported normal or corrected vision.

Apparatus and Procedure. The stimulus figure was always a perspective depiction of a $10.2 \times 10.2 \mathrm{~cm}$ black square, drawn to appear either as a square or as a diamond (see Figure 1). In the virtual space being depicted, the square's front and back edges were horizontal parallels to the picture plane; the diamond figure was formed from the square by rotating it (in virtual space) $45^{\circ}$ in its own plane about its own center, so that corners, rather than sides, were nearmost and farmost from the station point. (There was always a difference in virtual depth between "front" and "back" edges.)

The figure was drawn to appear either horizontal (flat) in virtual space, or else to be inclined upward from front to back-that is,
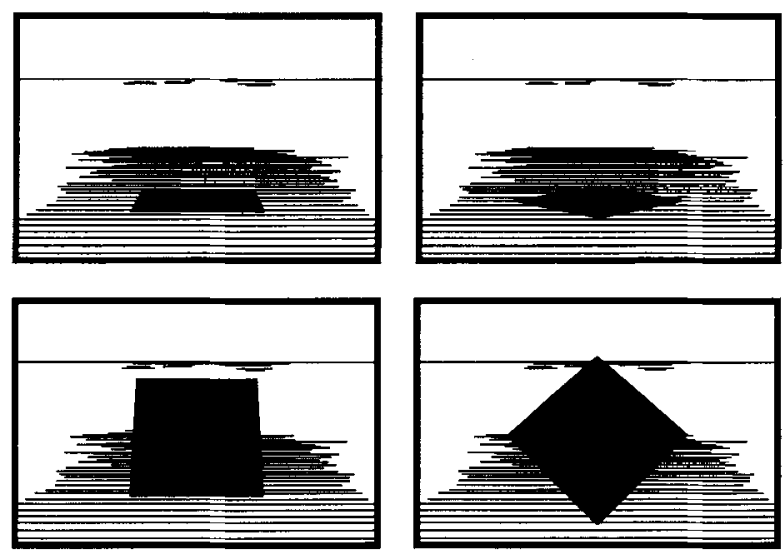

Figure 1. The four original pictures used in Experiment 1. They are, from left to right and then top to bottom, the flat square, flat diamond, pitched square, and pitched diamond. They were separately presented; each was viewed both frontally and from $60^{\circ}$ to the right. The figures were solid black, and the background hatchlines were unmerged, in the pictures as presented. 
from near to far-at $60^{\circ}$ to the virtual horizontal, on an implicit horizontal "hinge" at the figure's front, which hinge axis was normal to an original observer at the station point. (The hinge for both diamond and square was at the position in virtual space of the square's front edge, so that the center of either figure, when tipped up or when horizontal, would be at the same virtual-space position.) In this tipped, or pitched, position, the square's or diamond's depth into depicted space was effectively decreased to half the depth it had when it was horizontal.

Initially, four pictures were made, comprising views of the square and of the diamond, each in the horizontal and in the pitched-up condition. These will be called the original picnures in this experiment, and are shown in Figure 1. Each picture had a rectangular border, within which there was a depiction of ground (to be described) extending toward the horizon. The hinge point at the figure's front, described above, was $61 \mathrm{~cm}$ into virtual space from the station point, and centered $10.2 \mathrm{~cm}$ beneath the principal ray (which is a perpendicular from the station point to the picture). The picture plane was $45.7 \mathrm{~cm}$ from the station point. (Thus, the depiction approached 75\% of the figure's real full size. This choice of viewpoint produced clearly visible linear-perspective gradients at a moderate viewing distance, while the method of stimulus construction made it possible to know the depiction geometry precisely.) The original pictures, then, were of a flat square, a pitched square, a flat diamond, and a pitched diamond.

In each picture, a horizon line extended from border to border. A 1.5-mm black, rectangular border, $15.2 \mathrm{~cm}$ high $\times 22.9 \mathrm{~cm}$ wide, was horizontally centered on the square or diamond, with the horizon $3.8 \mathrm{~cm}$ below the border's top edge. A ground plane, $12.7 \mathrm{~cm}$ below the line of sight in virtual space, was represented by line segments that were selected from a pattern of parallels to the horizon, the parallels being $2.54 \mathrm{~cm}$ apart in the ground plane.

Four other pictures were also made. These had no frame, and no background except a horizon line. They were "second-order" reprojections of the flat and pitched squares and diamonds, as if these were seen from the side viewpoint that was actually being used with the original pictures. In each of these reprojections, as is shown in Figure 2, the horizon line was extended beyond what would have been the projection of the frame, to a length of $23 \mathrm{~cm}$, to prevent horizon length from becoming an unintended cue to the original picture-plane's orientation. These pictures will be referred to as redepicted flat square, redepicted pitched square, redepicted
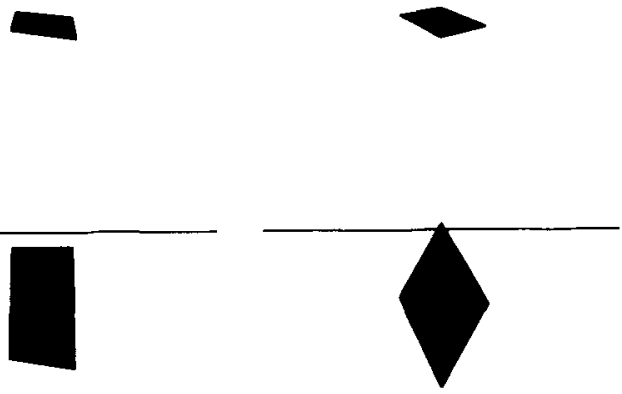

Figure 2. The four frameless stimulus pictures in Experiment 1. From left to right and then top to bottom, they are the redepicted flat square, redepicted pitched square, redepicted flat diamond, and redepicted pitched diamond. Exch depicts an original Experiment 1 figure, as seen from $60^{\circ}$ rightward. They were presented individually; their horizon lines each extend beyond where the original frame, in trapezoidal projection, would have cut them off. flat diamond, and redepicted pitched diamond, to distinguish them from the original pictures.

Four more reprojections were created and presented. They were two views each of the flat square and flat diamond, as if scen from aside. For each figure, there was a picture with both a redepicted frame and redepicted ground lines, and one with the frame (and horizon) only. These pictures had two potential purposes: (1) In case there was no rotation without figural context, they could show whether it was the absence of the frame or of the background that made the difference. (2) Judgments of frame orientation could be made, as well as estimates of whether that orientation was independent of intradepiction (background) contextual cues. Together, these four pictures will be referred to as ancillary stimuli, and individually they are named redepicted flat square plus frame (Figure 6, left), redepicted flat square plus frame plus ground (Figure 3), redepicted flat diamond plus frame, or redepicted flat diamond plus frame plus ground, as appropriate. (There were no ancillary pitched figures, out of consideration for the subjects' endurance.)

All pictures were ink-jet computer printouts on white bond paper, edge-mounted to white canvasboard, $22.9 \mathrm{~cm}$ high $\times 30.5 \mathrm{~cm}$ wide.

The 4 original pictures were each shown twice per subject, once to the side viewing position and once frontally. The 8 " as if aside" redepictions were shown once per subject, frontally. This made a total of 12 pictures displayed in 16 presentations per subject.

The pictures were mounted above a table, in a diffusely well-lit room. One subject at a time was carefully positioned at the table and was restored to this position as was necessary while the pictures were presented in succession, but was not restrained. When the subjects saw the original four pictures frontally, it was from $45.7 \mathrm{~cm}$, the distance of a canonical view-that is, a view from the station point. All other viewing was from a distance of $91.4 \mathrm{~cm}$ to the center of the drawn horizon. This distance was chosen to agree with the affine transform of the original station point to a position $60^{\circ}$ rightward, which was used to redepict the eight "as if from aside" pictures. (This change in viewing distance with viewpoint is also the change one would experience in walking along a wall on which a picture was hung.) The four original pictures, when shown from aside, were seen from $60^{\circ}$ to the right of a frontal view. The pictures' heights were adjusted for each subject, to put the horizon approximately at eye level. The picture plane was always vertical. Figure 3 shows how the original flat square would have appeared to a subject from aside, and how its redepiction would have appeared frontally.

As each picture was presented, the subject was asked to judge the horizontal direction in which the black figure appeared to be headed, from the subject's viewing position, and to adjust a heading indicator accordingly. The subjects were told to judge on the basis of the figure as a whole, not just selected features of it, and to consider the heading with reference to its deviation from their line of sight to the center of the picture.

Two distinctly different indicating devices were alternatively provided, each when appropriate. For depictions of the horizontal square or diamond, the indicator itself was also flat, on the surface of the table. A $10.2 \times 10.2 \mathrm{~cm}$ black square was fixed centrally on the surface of a $15-\mathrm{cm}$-diameter disk. The disk pivoted within the circumference of a larger, $25-\mathrm{cm}$-diameter white disk to which it was mounted. Beginning at the diameter of the smaller disk, light pencil lines extended about $4 \mathrm{~mm}$ outward on the surface of the larger disk, for use in recording the judged angles. The purpose of such an indicator was to avoid any explicit verbal reference by the experimenter to the figures as being square or diamond shaped, which reference might suggest some response strategy regarding edges versus corners as orientational markers. The subjects were instead told that the picture was of a horizontal, equal-sided figure "just like the one on the disk" on the table in front of them.

For the four ancillary flat-figure redepictions, which included a trapezoidal picture of the frame, a $29-\mathrm{cm}$ auxiliary pointer extended 


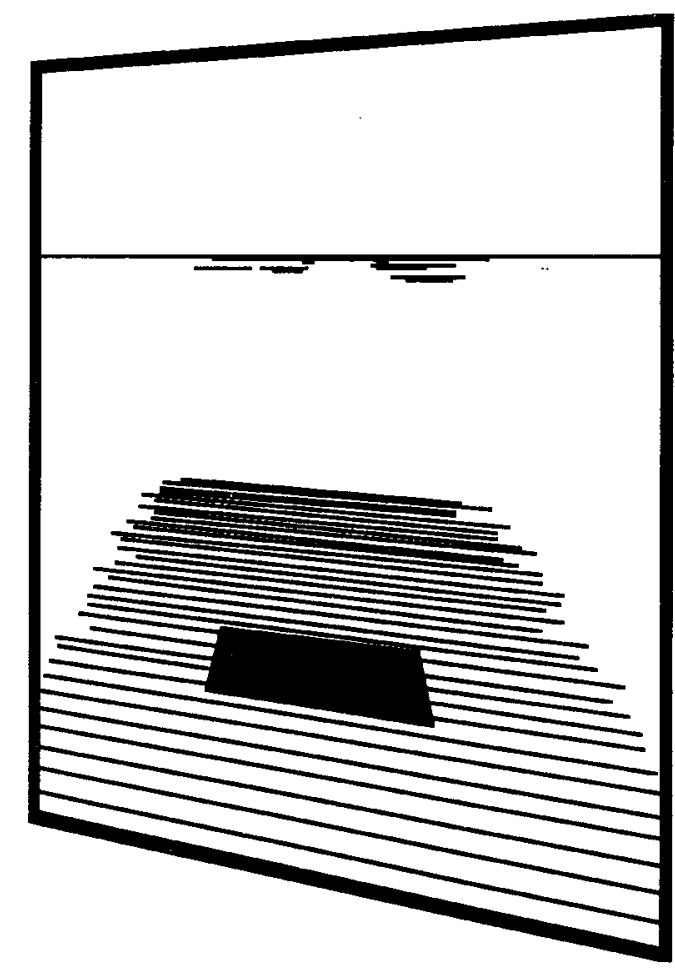

Figure 3. The redepicted flat square plus frame plus ground, Experiment 1 . It replicates the array from an original picture, the flat square, when that picture was seen from $60^{\circ}$ to the right of $a$ frontal view.

from beneath the indicating disk and was independently pivoted from the same center. The subjects were instructed to represent the orientation of the black figure by rotating the disk that had such a figure displayed on it, and to align the auxiliary pointer in the direction of the plane of the depicted frame, making both judgments with respect to their own line of sight.

In the pitched-figure presentations also, a full-sized black square/diamond was pivoted to indicate headings. But the indicator's square was in these cases inclined $60^{\circ}$ to the horizontal, on a $10.2-\mathrm{cm}$-tall vertical post, and was rotated within its plane by the experimenter at the start of a presentation, to appear either as a square or as a diamond (i.e., with either an edge or a corner downmost), for pitched-square or pitched-diamond pictures, respectively. The indicator's figure was thus rigged to have the same orientation in real space that the picture's figure had in canonical virtual space. The vertical post was rigidly attached to a horizontal pointer, which pivoted within the edge of the larger $(25-\mathrm{cm})$ white disk described above. The subjects were told that the inclined figure accurately represented what was drawn, and that they were to report rotation of the depiction's horizontal heading - that is, its orientation about a vertical axis rather than an inclined one.

Figure 4 shows the indicators for both flat and pitched figures. For all pictures, the larger disk was placed flat on the table, so that its center was $15 \mathrm{~cm}$ in front of the subject's eye position, and was approximately $50 \mathrm{~cm}$ below it, depending on the subject's sitting height.

Four subjects saw the redepictions before seeing the original pictures from aside; 3 saw the reverse. Following all other presentations, the four original pictures were shown frontally. (Care was taken that subjects not see the pictures frontally before making all other judgments.) Within this pattern of presentations, picture order was randomized.
Results

When the four original pictures were seen from aside, it is clear that differential rotation occurred; the horizontal figures rotated almost completely, and over twice as much as did the inclined ones, which instead were seen to be veering away toward the right. (See Figure 5.) Observed headings of squares did not differ appreciably from those of diamonds of the same inclination. A 2 (depths) $\times 2$ (shapes) $\times 7$ (subjects) repeated measures analysis of variance (ANOVA) showed significant effects of a change in virtual depth $[F(1,6)=80.91, p<.001]$, but not for shape (square vs. diamond) $[F(1,6)<1]$ nor for any interaction between shape and depicted depth $[F(1,27)$ $<1]$. The means in Figure 5 are amounts of counterclockwise (CCW) rotation (as if seen from above) from a heading perpendicular to the picture surface, which is the heading canonically depicted for all the objects. Throughout the study, full rotation to a real or simulated side view is $60^{\circ} \mathrm{CCW}$.

When the original pictures were in fact seen frontally, the figures were always (correctly) judged to be headed perpendicularly into the picture-that is, at $0^{\circ}$ rotation in each case. These frontal judgments are not represented in Figure 5.

Mean rotations expressed for the as-if-aside redepictions have the same meaning as for the original pictures, except that the picture's original position, which is the reference for rotations, was depicted instead of actual.

The frameless redepictions (Figure 2) rotated much as had their framed counterparts, except the frameless redepicted pitched diamond. The frameless squares exhibited nearly the same differential in rotation between flat and inclined ones as their array-equivalent squares in the sideviewed framed originals had. (See Figure 5.) A 2 (depths) $\times 2$ (frames) $\times 7$ (subjects) repeated measures ANOVA showed a significant effect of depth $[F(1,6)=36.16, p<$ $.001]$, but not of the frame's presence versus its absence $[F(1,6)=2.04, p>.10]$, nor was there any interaction of the frame's presence/absence with depicted depth $[F(1,27)<1]$.

The results from frameless redepictions of diamonds are less clear, however, because of an anomaly in the redepicted pitched diamond; evidently, it was perceived as multistable (Attneave, 1971). (The redepicted flat diamond was not;

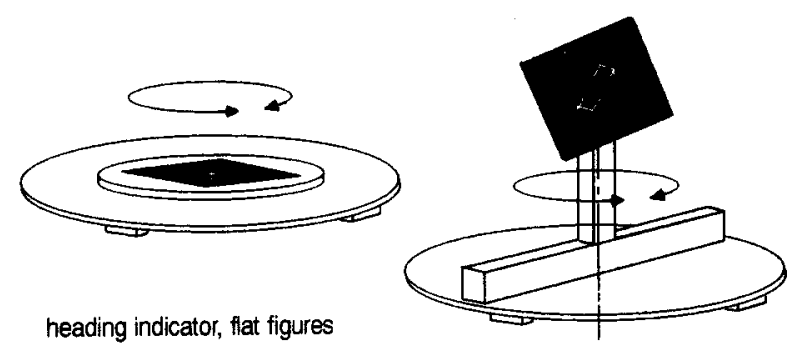

heading indicator, pitched figures

Figure 4. Left to right, the indicators for flat-figure and for pitched-figure heading judgments in Experiment 1. 


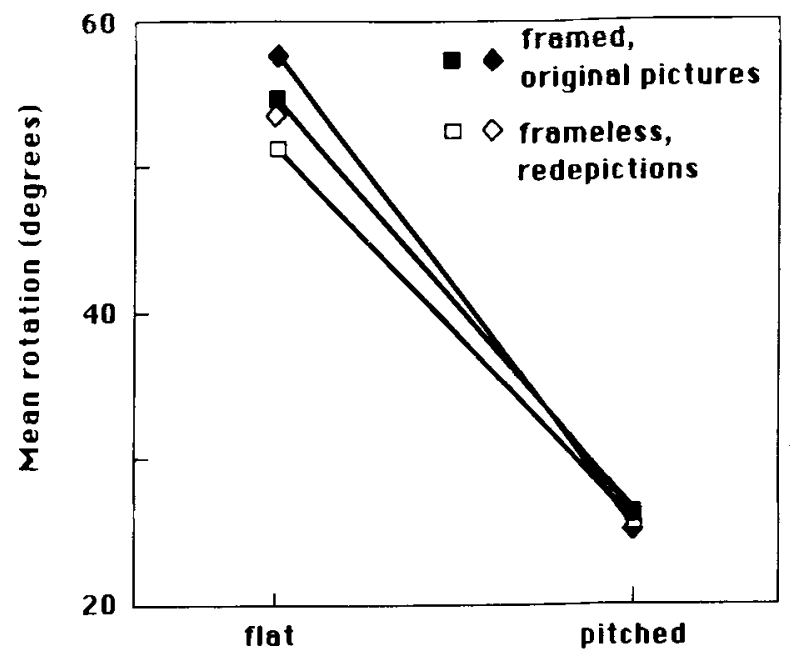

Depicted attitude of square or diamond

Figure 5. Selected mean judged headings of depicted figures in Experiment 1. The judgments of original-picture figures, seen from $\mathbf{6 0}^{\circ}$ aside, are compared with judgments of the frameless secondorder redepictions of the squares. Seen frontally, the redepicted squares matched the array projections of the original-picture squares to the $60^{\circ}$ rightward viewpoint. The headings are expressed as amounts of rotation counterclockwise from a perpendicular through the picture plane in its depicted position. Standard errors of the heading means were these: For original-picture judgments, they were, for the flat square, $3.3^{\circ}$; flat diamond, $1.8^{\circ}$; pitched square, $5.4^{\circ}$; and pitched diamond, $4.9^{\circ}$. For judgments of frameless redepictions, they were, for the flat square, $1.8^{\circ}$; flat diamond, $3.2^{\circ}$; and pitched square, $6.1^{\circ}$. The redepicted pitched diamond data are omitted because that figure was multistable (Attneave, 1971).

it exhibited nearly the same rotation as did the redepicted flat square and as the two original-picture horizontal figures.) It is apparent in Figure 2 that leftward and rightward orientations for this figure might readily alternate, and 3 subjects reported that they did. These subjects and 1 other, however, saw $\mathrm{CCW}$ rotations as usual. The 3 remaining subjects did not report reversals, but judged the pitched diamonds to be headed left (that is, to rotate clockwise [CW]), instead of right (CCW) as depicted and as all subjects saw for the same geometric array when framed, and in all other real or simulated side viewing, of framed or unframed pictures. If the direction of rotation were ignored, and only its absolute amount were considered, the mean rotation of the redepicted pitched diamond could be reported as $26.6^{\circ}$, an amount indistinguishable from mean rotations for the other pitched figures. However, the result for this picture is sufficiently inconclusive that it is omitted from the means for pitched figures that are given in Figure 5.

The four ancillary redepictions, all with horizontal figures and all framed, rotated strongly as expected. Their mean rotations were as follows: redepicted flat square plus frame plus ground, $55.2^{\circ}$; redepicted flat diamond plus frame plus ground, $57.0^{\circ}$; redepicted flat square plus frame (no ground), $46.9^{\circ}$; redepicted flat diamond plus frame (no ground), $56.6^{\circ}$. The standard errors of these mean ro- tations were, respectively, $2.6^{\circ}, 3.1^{\circ}, 4.3^{\circ}$, and $3.6^{\circ}$. Despite the seeming decrement in rotation for the horizontal square without ground lines (which is unexplained), a 2 (shapes) $\times 2$ (backgrounds) $\times 7$ (subjects) repeated measures ANOVA showed no significant effect of square versus diamond shape $[F(1,6)=5.19, p>.05]$ or of whether the background was present or absent $[F(1,6)=1.26, p>$ $.10]$, nor was there any interaction of shape with the background's presence/absence $[F(1,27)=1.28, p>.10]$. The effect on rotation, of having background lines that might visually link the figure with its frame, is minimal or nonexistent, then, in comparison with the strong effect of depicted figural orientation that is evident in other parts of this experiment.

The depicted frames rotated also, by a consistent amount that was less than that for the figures. Frame rotation is expressed as CCW rotation from the plane depicted for it to the plane in which it was judged to lie, as indicated with the pointer. Mean frame rotations, in the order that the pictures were listed in the previous paragraph, were $35.9^{\circ}, 35.5^{\circ}, 33.4^{\circ}$, and $36.8^{\circ}$; standard errors of these means were $4.6^{\circ}, 2.5^{\circ}, 4.4^{\circ}$, and $2.0^{\circ}$, respectively. These frame rotations were distinctly different from the figure rotations in the same pictures. A 2 (features: figure or frame) $\times 4$ (pictures) $\times 7$ (subjects) repeated measures ANOVA showed a difference between figure and frame rotation $[F(1,6)=25.55, p<.005]$, but no significant difference in rotational characteristics from picture to picture $[F(3,18)=3.06, p>.05]$, nor was there any interaction of figure-versus-frame difference, from picture to picture $[F(3,55)<1]$.

\section{Discussion}

Horizontal figures invariably rotated much farther than did the pitched ones, except that the multistable frameless pitched diamond was unmeasurable in this regard. Thus a depicted object's virtual depth, or something here indistinguishable from that depth, would seem to encourage object rotation.

The alignment of the object's contour in virtual space, to form a square or a diamond, did not seem to affect rotation, except that of the multistable redepicted pitched diamond. If edge orientation does not influence object rotation, that might suggest that the object's particular shape does not matter either, except as that would bear on its depth proportion in virtual space. It seems to have been something about their different proportion in length, for example, that caused rods and disks of the same axial orientation to rotate differently in Goldstein (1979). When their axes were nearly parallel with the picture surface, that was also the direction of the rod's extent, but the disk's largest dimension was in depth, which should have made it rotate more, as it did.

Whatever the real, fundamental cause of rotation, it almost certainly exists in the depiction of the object itself, with no necessary dependence on picture-plane or framing cues, since a robust rotation occurred in their absence. This means that, whatever the role of the perspective taper 
of horizontals across a picture toward a side vanishing point, in specifying the orientation in space of directions widthwise across the picture, that role does not depend on specifying a common direction of such objects with the frame. It does not mean, however, that when information for such commonality does exist, it cannot be additionally effective, especially for those "depthless" objects lying along the picture plane. This issue will be taken up in Experiments 4 and 5.

It is difficult to separate putative rotational factors within a depiction itself, and object depth was imperfectly isolated. The problem is partly that a single depictive element-a contour, perhaps, or a dimension-may contribute to several different (and sometimes disparate) geometric indicators of the depicted object's orientation that are present (Halloran, 1989). It therefore is not certain that virtual depth was the operative rotational factor here. A conspicuous confound is depicted object height, which varied inversely with depth in these pictures. It could have been the pitched figures' taller heights that caused them to rotate less: Figural slimness, produced by compressive reduction in figural width, to the oblique viewpoint, specifies a heading that is angled aside from the line of sight. That reduction in width may have been more evident and salient in the taller, pitched figures, even though its amount was exactly the same as in the horizontal figures. But the case for object height as a generally important factor in rotation is doubtful. Goldstein $(1979,1987)$ repeatedly demonstrated rotational differentials among object depictions that did not differ in height at all. Furthermore, when Ellis, Smith, and McGreevy (1987) specifically investigated the rotations of depictions of pointers of two heights (flat and inclined pointers), there was virtually no effect of pointer height (the inclined pointer was taller) when the pointers were depicted at $60^{\circ}$ or, comparable to the case of squares and diamonds here, perpendicular $\left(90^{\circ}\right)$ to the picture plane. (They did, however, find that the greater height had less rotation when the pointers were depicted in headings parallel to the picture plane or at $30^{\circ}$ to it. To the extent that this interaction of height with depicted orientation is generalizable to the present case, it would suggest that height was not a factor in the present square/diamond experiments.)

Goldstein (1987) studied differential rotations in pictures showing different directions of gaze, and discussed these as instances of rotation that did not materially involve object depth. It is true that the iris of a human eye has almost no depth in virtual space. But surely the gaze has the implied depth, in Gombrich's (1978) meaning, of all the distance from its origin to its object. This distance is indefinite when its object is neither depicted nor otherwise known (it is known, for example, when it is the observer), but it is not small. Goldstein's results, in fact, correspond fairly well with a depth/width-ratio interpretation. Gaze rotates more when it is straight out of the picture and its virtual width is zero, than when it is sidewise with large implied width.
Although object rotation does not depend on contextual information about the picture surface's location or about the object's scenic placement, it is clear from the multistable redepicted pitched diamond that contextual cues can sometimes help define the depicted object's general direction and can in that way contribute to an informed rotational judgment.

The redepicted frames (around ancillary figures) rotated, and by amounts roughly comparable with some depicted figures in other pictures in the experiment. The remainder of this study is largely focused on whether and how this frame rotation could affect picture perception. Two immediate questions arise: Though objects rotate independently of their frames, is the reverse also true? Also, do real frames rotate, not just depicted ones?

\section{EXPERIMENT 2}

In order to demonstrate whether frame rotation is independent of object rotation within it, as-if-from-aside reprojections were used, as in Experiment 1. Here, they were framed versions of both the horizontal and the pitched squares (Figure 6). The squares in the two pictures have been shown to rotate quite differently from each other, in Experiment 1; if the frame rotation is independent of this difference, the frames of the two pictures should rotate alike.

\section{Method}

Subjects. These were 7 female and 4 male students of the University of Maryland, Asian Division, who were taking undergraduate courses at Yongsan Army Post, Seoul, Korea. Six of these were members of a class in social psychology being taught by the author; 4 others were members of the University of Maryland's Yongsan administrative staff, as well as being students. Ages ranged from 17 to 61 years; their median age was 24 years. All reported normal or corrected vision. None had participated in the previous experiment.
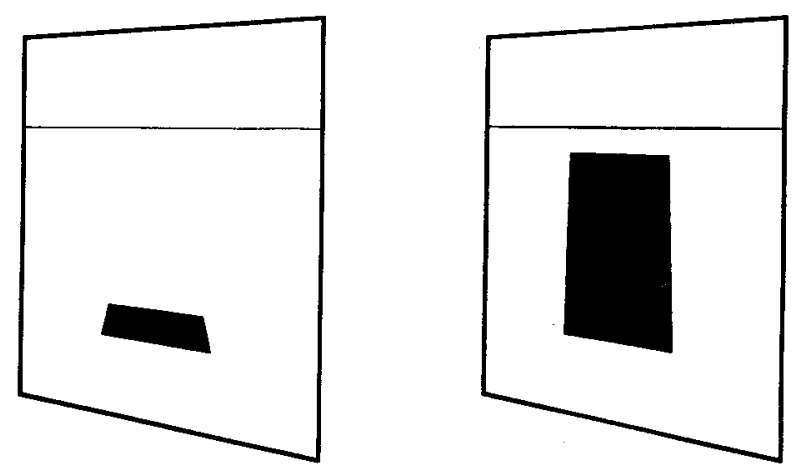

Figure 6. The stimulus pictures of Experiment 2, presented frontally. Left to right, they are the redepicted flat square plus frame and the redepicted pitched square plus frame. They each represent the figure and frame of an original picture from Experiment 1 , as those features would appear when seen from $60^{\circ}$ to the right. The flat-square picture (left) was also an ancillary picture in Experiment 1. 
Apparatus and Procedure. Two original pictures from Experiment 1 , the flat square and the pitched square, were reprojected to be, respectively, a redepicted flat square plus frame and a redepicted pitched square plus frame, in the manner described in Experiment 1. This is the array equivalent of seeing the originalpicture squares and their frames from $60^{\circ}$ rightward of the station point, and from a $91.4-\mathrm{cm}$ distance. Viewing conditions and procedure were the same as for redepictions in Experiment 1, except for the configuration of the pointer/indicator used to specify angular judgments. In light of a reviewer's concern that the pointer of Experiment 1, because it resembled the figure being judged, might in some way affect the subjects' judgments, a simpler indicator was used. This was a flat, $29-\mathrm{cm}$ pointer that would pivot about its center on a flat circular base. The base was placed in the same position as in Experiment 1. (This indicator was identical with the one in Experiment 4 in Halloran [1989], except that the previous version employed two concentric $29-\mathrm{cm}$ pointers.) A full-size cardboard replica of the square, in the appropriate (horizontal or pitched) position, was shown to subjects before, but not during, each picture presentation.

For each picture, the subjects indicated two directions, relative to their line of sight to the picture: the horizontal direction (heading) toward which the square appeared to extend in depth, and then the horizontal angle at which the frame seemed to be placed. (If the judgment order had been reversed, some subjects might have referenced their object orientations to the picture surface, instead of to the sightline, and confused the results. Experiments 4 and 5 consider this issue.) Picture order was counterbalanced.

\section{Results}

Mean rotation of the flat square was $55.5^{\circ}$; of the pitched square, $27.1^{\circ}$. The mean rotations of their frames, respectively, were $25.5^{\circ}$ and $29.6^{\circ}$. In the same order, standard errors of these four means were, squares, $2.0^{\circ}$ and $4.6^{\circ}$; frames, $2.9^{\circ}$ and $3.5^{\circ}$. Here, as in Experiment 1 , mean rotations are expressed as amounts of $\mathrm{CCW}$ rotation from a heading perpendicular to the surface which is being simulated in the redepiction. As expected, the flat square rotated much more than did the pitched one $[F(1,10)=41.76, p<.001]$. The two mean frame rotations did not differ from each other or from the mean rotation of the pitched square $[F(2,20)<1]$. The mean frame rotations were each nonzero: for the flat-square frame, one-tail $t(10)=8.73, p<.0005$; for the pitchedsquare frame, one-tail $t(10)=8.53, p<.0005$.

\section{Discussion}

The frame rotates by the same amount regardless of its depiction's rotation, which seems to establish their independence in this respect. This does not necessarily mean that frames of all shapes will rotate alike. It also does not guarantee that a real frame will rotate. The real frame has extent in real depth, not virtual depth, and it does not quite have constant aspect, though a border may have that, and many frames nearly do. Experiment 3 will examine a frame with real depth.

\section{EXPERIMENT 3}

Perhaps depicted frames only rotate because this is something that pictured things do. A real frame might not, since there is more information present with which to lo- cate it precisely-for example, in the angles of the picture's wall with the floor and ceiling, a visible edge, shadows, shading, and sheen.

\section{Method}

Subjects. There were 23 subjects. Twenty-one, including 10 enlisted soldiers in the United States Army, were undergraduate students attending the University of Maryland, Asian Division, at Yongsan (Seoul), Korea; of these, 12 were enrolled in an introductory psychology course, and 9 were in a research methods course, at the time. There was also 1 member of the University of Maryland's local administrative staff, and 1 Lecturer in English for the University. Of the total, 12 were male. Ages ranged from 17 years to 52 years; the median age was 22 years. All subjects reported normal or corrected vision. None had participated in the previous experiments.

Apparatus and Procedure. The stimulus picture was a $14.0 \times$ $22.1 \mathrm{~cm}$ printed monochrome reproduction of Winslow Homer's The Fog Warning, mounted flush to a medium brown, $20.8 \times$ $29.8 \mathrm{~cm}$ framing surface. The surface had a clearly visible, $0.5-\mathrm{cm}$ thick outer edge. There was no appreciable gloss in either the picture or the framing surface. The picture represented a rowboat at sea. In depicted space, the boat was headed approximately $22^{\circ}$ leftward of a straightaway orientation from an observer of the picture, according to a projective reconstruction of the pictured space, described in Halloran (1989; see especially Figures 4 and 6 therein). The subjects, one at a time, sat at a standard office desk directly before the picture, which was shown in two ways, obliquely and frontally. The oblique picture was placed to be seen from a $60^{\circ}$ rightward viewpoint, as done previously for side viewing; the viewing distance was $81.5 \mathrm{~cm}$ along the line of sight to the center of the picture. The distance to the frontal picture was $40.7 \mathrm{~cm}$. In both cases, the picture was vertical, mounted on a platform above the desk-surface level, to place the horizon approximately at eye height for all subjects. The picture, other apparatus, viewing angle, and viewing distance were virtually the same as those in Halloran (1989, Experiment 4), except in three respects: The picture size and viewing distance here were reduced by $3 \%$ (in each dimension) to take advantage of a superior reproduction that was available, the frame was brown, and the subjects saw the picture frontally as well as from aside. The pointer was that from Experiment 2, positioned as before.

At each orientation of the picture, the subjects used the pointer to express their judgments of the horizontal angle, or heading, of the rowboat with respect to their own line of sight toward it, and then to judge the horizontal direction of the picture's border, also with respect to the line of sight. Order of picture orientation was counterbalanced.

\section{Results}

Frame rotation is reported as the amount by which the frame seemed more nearly frontal to the line of sight than it really was. Mean rotation of the oblique frame was $13.5^{\circ} \mathrm{CCW}$, with a standard error of $3.0^{\circ}$. This rotation was significantly nonzero $[t(22)=4.55, p<.0005$, onetail], and definite CCW rotation was seen by all but 3 subjects. All subjects judged the frontally presented frame to be exactly frontal.

Boat orientation with respect to the line of sight did not appreciably differ between oblique and frontal viewing $[F(1,22)=1.67, p>.10]$. Mean judged boat headings (not rotations) were $21.7^{\circ}$ leftward of straightaway when viewing was oblique, and were $24.1^{\circ}$ leftward of straightaway when the picture was seen frontally. Standard er- 
rors of these means were $1.92^{\circ}$ and $2.67^{\circ}$, respectively. At both viewpoints, all subjects saw the boat to be heading leftward.

\section{Discussion}

This real frame rotated with viewpoint, despite the presence of shading and shadow, and despite the fact that surface intersections beneath the frame might have revealed its veridical location with some accuracy. Not enough is known about the causes of rotations, however, to make it certain that these other factors do conflict perceptually with the frame's observed rotation.

The boat-heading judgments from both positions compare favorably with the $22^{\circ}$ leftward heading that Homer seems to have depicted (Halloran, 1989). Projective compression (Goldstein, 1987) might have reduced the amount by which the obliquely viewed rowboat seemed to head left of the line of sight, but there was little or no such effect.

Nonetheless, the obliquely judged boat had virtually full rotation toward the viewer. This result is what one could expect if the array itself, and no other factors, determined the perception of orientation in pictures (Cutting, 1988; Halloran, 1989). (Despite compression, an array-determined boat must appear to head leftward.)

But the objects and their frames both rotate. It follows that when subjects are asked (in effect) to subtract the frame rotation, by judging object orientation from the picture plane, the rotation that is seen and reported should be substantially less than full. That in fact is the consistent result in Halloran (1989, Experiment 3), where the boat's rotation from its picture plane tended to be judged as approximately $50 \%$ of full, versus virtually $100 \%$ in Experiment 6 of that study, and also in the present experiment, with rotation measured in both latter cases simply as a turning toward the subject. (The rotations in both studies have been observed with arrays that were geometrically identical, although absolute scale and distance of the picture, and chromaticity, have differed.) Nonetheless, it should be shown directly that object and frame rotations can be subtracted.

\section{EXPERIMENT 4}

This and the following experiment have had two purposes: to show directly that frame rotation sometimes affects judgments of object orientation, depending on what kind of judgment is being made, and to examine some properties of a class of pictures in which the objects are "depthless"; that is, they appear to lie in or parallel with the picture plane. (Real objects have some thickness and therefore cannot be truly depthless. Nonetheless, the depth component of their orientation in virtual space can be zero, and it often is.)

Indirect evidence discussed in Experiment 3 suggests that frame rotation can reduce a depiction's rotation relative to its surface. A strong prediction from this would be that object rotation (to the line of sight) minus frame rotation (to the line of sight) equals object rotation (to the apparent picture surface). To test this, subjects in Experiment 4 judged rotations of both a depicted object and its frame, separately. Then the different subjects in Experiment 5 judged the "difference," the amount by which the object rotated from its framed surface.

Depthless objects ought not to rotate at all, if a strict depth/width ratio is the sole factor. Yet a measureable, perhaps residual, rotation seems to remain, even for pictures with hardly any depth (Goldstein, 1979). This suggests that an additional factor in object rotation is effective regardless of depth. The depiction's length along the picture surface is a candidate factor. (Whether that extent would commonly be called a length or a width might depend on the depicted orientation. In a canonical view, it has the same size as virtual width; otherwise, it may not.) As explained in Experiment 1, when an object extends flatly across the picture, that orientational alignment with the surface is apt to be specified in the object's projection, in perspective gradients. For this reason, and/or because such depictions do not rotate much in comparison with others, Goldstein $(1979,1987)$ and Halloran (1989) have suggested that an object's extent parallel with the picture plane might actively prevent its rotation, in some way. If so, two things follow: (1) The effect of horizontal flatwise extent per se will be maximal with depthless depictions (which proportionately have the greatest flat extent), and (2) the amount of that flat extent might be differentially effective (whether because of greater length of perspective gradient, relative proximity to the picture's edge for visual reference, or something else). Therefore, five different lengths of depthless object were investigated.

If object and frame rotations are truly independent, depthlessness in the object should not affect frame rotation, even though depthlessness is at one limit of orientation, provided only that depthless objects exhibit rotation. Objects rotate with or without visible frames (Experiment 1), and, conversely, large differences in object rotation do not affect frame rotation (Experiment 2); therefore, the two seem to be independent. Even if a depiction's rotation were found to be affected by its relative extent from border to border, there is no particular effect of this on frame rotations, that could be predicted. But it will be seen whether there is any substantial dependence of rotation on surface extent, to be worried about at all in this regard.

\section{Method}

Subjects. These were 8 students in an extramural graduate program in systems management that was jointly offerred by the University of Denver and the University of Southern California. Five were United States Navy commissioned officers, of whom 2 were aircraft pilots. The 3 civilians were an engineer and 2 systems analysts. Ages ranged from 28 to 40 years; 4 subjects were female. All subjects had normal or corrected vision. None had participated in any of the previous experiments.

Apparatus and Procedure. The stimuli were pictures of doublepointed arrows of five lengths: $7.6,11.4,15.2,19.1$, and $22.9 \mathrm{~cm}$, drawn with black lines on a white surface, and centered horizontally within a 1.5 -mm-thick black border that enclosed a surface $17.5 \mathrm{~cm}$ high $\times 25.1 \mathrm{~cm}$ wide, and was in turn centered on the 
overall picture surface, which was $22.9 \mathrm{~cm}$ high $\times 30.5 \mathrm{~cm}$ wide (See Figure 7.)

One at a time, the subjects sat at a table to view the pictures. These were in front of the subject and perpendicular to the table on a platform above it; platform height was adjusted to place the arrow approximately at eye height for each subject. The picture plane was always $30^{\circ}$ from the line of sight, so as to be seen from $60^{\circ}$ to the right of a frontal view. The viewing distance was $76 \mathrm{~cm}$ to the picture's center. The pictures were presented in random order. The subjects were asked to judge the horizontal direction of the arrow and also of the picture itself. The directions were to be judged relative to the line of sight through the center of the picture. The judgments were indicated with a pair of $29-\mathrm{cm}$ pointers of different colors that pivoted about a common center on the large disk used in Experiment 1, which was set on the table as in that experiment.

(The pictures were not viewed frontally, for practical reasons. Though it might have been desirable to do, none of the headingjudgment comparisons made here and in the next experiment involved or required frontal-viewing data. In any case, the frontal appearance of these pictures [Figure 7] does not seem doubtful.)

\section{Results}

For both the arrow and the picture frame itself, judged orientations are expressed as $\mathrm{CCW}$ rotations from the real orientation of the picture surface, which in this case was coincident with the plane of (the arrow's) depiction. Mean arrow rotation was $33.4^{\circ}$; mean frame rotation was $20.6^{\circ}$.

There was no apparent effect of arrow length on rotation. A 2 (rotations: arrow or frame) $\times 5$ (lengths of arrow) $\times 8$ (subjects) repeated measures ANOVA showed a difference between arrow rotation and frame rotation $[F(1,7)=22.78, p<.005]$, but no significant difference among arrow lengths $[F(4,28)<1]$ nor any interaction of the difference between frame and arrow rotations with arrow length $[F(4,79)<1]$.

As in Experiment 3, the frame rotation was clearly nonzero $[t(7)=10.45, p<.0005$, one-tail $]$. The mean rotations of arrow and frame at each arrow length are shown in Figure 8.

\section{Discussion}

The object rotations in this experiment did not require any depicted depth, and were unrelated to picture-surface

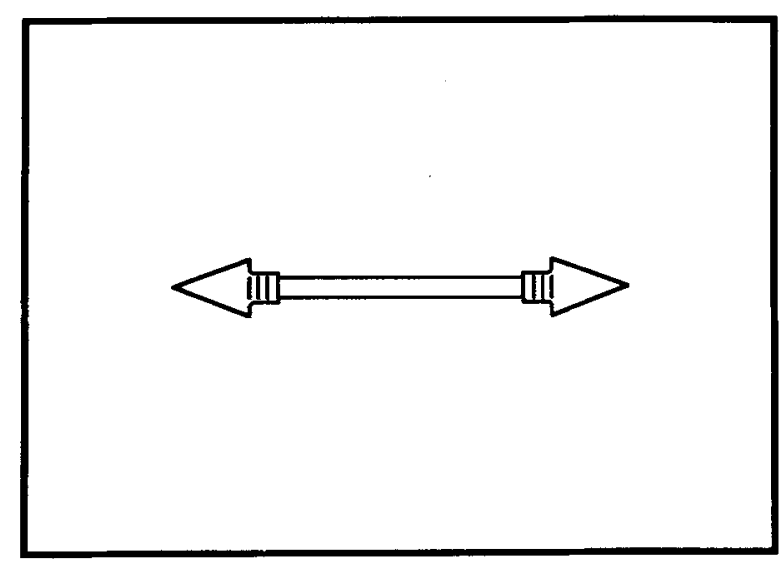

Figure 7. The 15.2-cm arrow picture in Experiments 4 and 5.

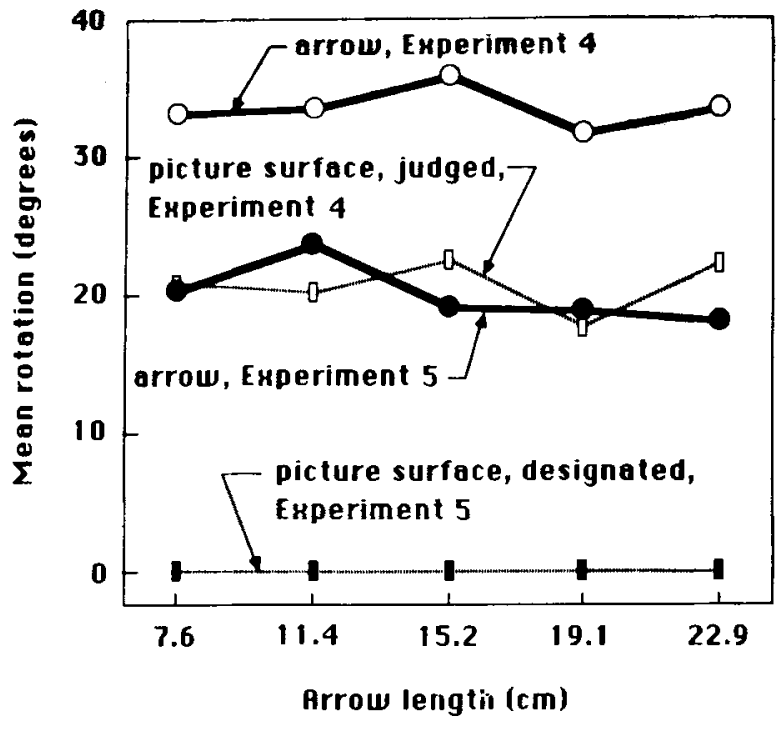

Figure 8. Means of the judged directions of the arrows and of the picture in Experiment 4, and of the arrow in Experiment 5. The directions are expressed as amounts of counterclockwise rotation from the angle at which the picture was actually presented. In Experiment 5, a pointer was preset to indicate to subjects that the picture surface was actually located in the position designated here as $\theta^{\circ}$ rotation. A full rotation to the observer's frontal plane would have been $60^{\circ}$ counterclockwise.

knowledge in any known way. It would appear that object depth is not required for the weakest rotations, but is required to produce stronger ones, as of the horizontal figures in Experiments 1 and 2. Whether there is some minimum depth that would act rotationally as no depth at all is unknown. Either there are at least two rotational factors, one linked with depth and one not, or else there is a single complex one. Whether regular distortion in the perception of visual extents (Higashiyama, 1992) might be a depth-free factor, and/or whether constant aspect could be a complex one, will be topics in the General Discussion.

It did not matter how far the arrow stretched across the picture. While some unspecified kind of interdependence might still exist, rotation of depthless figures does not depend on their extent, neither absolutely nor as a proportion of picture width, in any way measurable here. This was expected, since figure and frame rotations are generally independent (Experiment 2), but it counters speculation (Goldstein, 1979, 1987; Halloran, 1989) that flatwise extent interacts with surface knowledge. The speculation had at least two bases: If an object's distance and length are known, its relative surface extent does trigonometrically specify its orientation; maximum extent specifies a length parallel to the surface. Alternatively, by a looser calculus, an extent that fills the frame might be seen as more probably coplanar than shorter lengths would be. But no effect of length was found that would need to be explained in either way.

Arrows turned farther than frames, even though the arrows should have had a minimum amount of object rota- 
tion (Goldstein, 1979). It seems unlikely, then, that frame rotation will often, if ever, exceed that of the depiction within. It is not necessarily true, however, that all depictions will rotate more than any frames. In Experiment 1, the redepicted frames rotated farther (mean of four frames, $35.4^{\circ}$ ) than did the original-picture pitched-up figures (mean of two figures, $25.7^{\circ}$ ).

\section{EXPERIMENT 5}

Using the same pictures as in Experiment 4, subjects specifically judged the arrows' rotations in relation to the picture plane. If surface information (including that in the frame) can be used to make such comparison, these judgments should reflect the difference between independent judgments of arrow rotation and of frame rotation that were made in Experiment 4.

\section{Method}

Subjects. These were 8 students in the previously mentioned University of Denver/University of Southern California graduate program in systems management, who had not participated in any other of these experiments. Four were United States Navy commissioned officers, of whom 3 were aircraft pilots. The civilian subjects were 2 engineers and 2 managers. Their ages were from 24 to 47 years. All subjects were male, and had normal or corrected vision.

Apparatus and Procedure. The apparatus was the same as in Experiment 4 . The procedure was also the same as in that experiment, except that one pointer was preset and a different instruction was given. Before presentations, the experimenter positioned one of the pointers in a direction $30^{\circ}$ counterclockwise to a subject's line of sight through the center of the picture, and the subject was told, as was the case, that this was the position in real space of the picture surface. The subjects were each asked to judge, with the remaining, unset pointer, the horizontal direction of the arrow with reference to the picture surface.

\section{Results}

Judged orientations are expressed as in Experiment 4. Overall mean arrow rotation was $19.9^{\circ}$. This rotation was nonzero $[t(7)=4.69, p<.005$, one-tail $]$. As in Experiment 4 , arrow length did not affect rotation $[F(4,28)<1]$. The mean rotations at each arrow length are shown in Figure 8.

When the mean of each subject's five judgments of arrow rotation (one for each length) were compared between Experiments 4 and 5, the rotations in this experiment were less than those judged independently of the frame in Experiment $4[F(1,14)=7.17, p<.025]$.

\section{Discussion}

Object rotation is evidently greater when judged directly as a turning toward the observer in Experiment 4 than when it is judged and expressed here as an amount of turning from the picture plane. The same kind of difference also seems to exist when data in this study are compared with certain results in Halloran (1989), as mentioned. Each instance of difference can be explained roughly as a subtractive effect of frame rotation, although the fit with the equation, object rotation (to the observer) minus frame rotation equals object rotation from the frame, is inexact. (See Figure 8.) Further work should be done to quantify this effect.

Considering the present result together with Experiment 1 , it seems clear that surface knowledge only affects object rotation when the surface is explicitly or implicitly an object-rotational coordinate.

Arrow length had no differential effect on rotation. This reinforces the identical finding in Experiment 4 and the argument against any directly acting, residual effect of surface awareness on depthless-figure rotation.

The prepositioned pointer was set at the veridical location of the picture plane, which, it is known from Experiments 3 and 4 , is not the plane at which the picture would be seen. This way of preindicating the picture surface was intended to be conservative, in that if subjects disregarded the instruction, and considered only the arrow's apparent direction in space (relative to themselves, as was proper for them to do in Experiment 4), that should have increased the amount of arrow rotation expressed here, and therefore reduced the difference in arrow rotation found between Experiments 4 and 5.

\section{GENERAL DISCUSSION}

This work confirms the theory that picture perception is array-determined, which has been proposed in various forms (Cutting, 1988; Gogel, 1990; Halloran, 1989). Further, it explains a controversy regarding surface awareness: With respect to orienting things in pictures, it seems that the main value of knowing the surface's location is to be able to relate the perceived surface slant to the perceived object slant. The object slant itself, with respect to the observer's space, ordinarily remains unchanged by the surface knowledge, contrary to previous supposition and/or speculation in Cutting (1988), Goldstein (1979, 1987, 1988), Halloran (1989), Kubovy (1986), Perkins (1973), Pirenne (1970), and Rosinski and Farber (1980).

A four-point summary of the findings may be given:

1. A depicted object's apparent orientation in real space, with reference to the observer, depends on characteristics of its own projective array.

Whether it always is the case, rotation here was differentially related to depicted virtual depth; deeper objects rotated more. However, even depthless objects rotated a distinct amount, so it seems likely that factors both related and unrelated to depth are involved. (Given the array-directed nature of the peception, it is more probable that virtual depth from the viewpoint of the moment, rather than the depth canonically depicted, determined the rotations. But momentary depth could not have been specified, because of multiple cues [Halloran, 1989].)

2 . Ordinarily, knowledge of the depicted object's surface has little or no direct effect on the apparent orientation that the object will have. If the object is multistable, however, and probably in other instances in which the perspective information that it yields is impoverished, the ability to locate the picture surface may help orient the object and stabilize it. 
3. The frame also rotates; that is, it is seen at less of a slant to the observer than it really has (Experiments 3 and 4) or than is depicted for it (Experiments 1 and 2).

4. Object rotation and frame rotation are essentially independent of each other, if these are understood as amounts of turning toward an observer's position. However, it is often the difference between the two rotations that matters, for example, when one is trying to decide the particular view that the artist or photographer had of the scene represented. (This is the question of what the picture really is like, rather than of where things in it seem to be going at the moment.) In the cases in which the rotations of object and frame are both attended to, they seem to subtract from each other. The difference between them may be small, in the case of side views or other depictions which do not rotate fully.

This subtractive effect of the frame rotation tends to stabilize the surface-to-depiction orientation over viewpoints, and in that way doubtless contributes to the general impression that things in pictures do not visibly distort much as the observer moves around (Cutting, 1987; Kubovy, 1986; Pirenne, 1970; Rosinski \& Farber, 1980). However, the corrective effect of the frame has nothing to do with differential rotation, which needs explaining in regard to this "unseen" distortion: If parts of a scene rotate farther than other parts, that ought to warp the scene being viewed. Yet scene-layout stability and differential rotation are commonly found in the same picture (Goldstein, 1979, 1987). Perhaps the departures from scene rigidity remain unnoticeably small (Cutting, 1987) because all parts of a depiction rotate together, even though some turn more than others (Goldstein, 1979). The warpage thus might be subthreshold or fall below some cognitive criterion for empirical relevance, as Busey, Brady, and Cutting (1990) suggest.

The conclusions of Busey et al. (1990) are only partially in agreement with what has been found here. They used pictures of faces, some of which had been slanted and then reprojected to remove valid surface information, and trapezoidal projections of frames which sometimes did not match the faces' projected slant. They did not find frame slant effective in the deciding of how much distortion was in the pictures. This would have beeen expected in the results here, if knowedge of the face's true orientation would aid in the evaluation of its distortion, since frame slant does not influence absolute orientation of the picture. However, Busey et al. also found that subjects could not match face slant with frame slant, and from this they concluded that people have "little ability to use frame slant information in slanted picture perception." This is disturbing, since the subtractivity of perceived frame slant from perceived object slant clearly implies the ability to compare the two. The faces in Busey et al., though, may not have been adequate stimuli for testing this ability to compare. The general ability of observers to tell whether a rectangular frame is slanted left or right is beyond question. However, that is not true of the faces; Busey et al. concluded that the face distortions were either subthreshold or "within the bounds of acceptability." A look at the faces in their Figures 2 and 5 suggests that this makes sense. The distortions from side to side are very subtle in comparison with the large amount of horizontal compression that is equally evident on either side. And anyway, faces in nature are not so regular that these small distortions in projection might easily be interpreted as evidence for orientation. (The problem is similar to that of the pitched diamond in Experiment 1, which was multistable when unframed and for which a reverse-slant frame probably would have yielded opposite rotations, though this was not tried.) The belief here, then, is that Busey et al. failed to find an ability to compare because their subjects could not readily orient the faces, not because they were unable to use framing information in the comparison.

Some researchers (Kubovy, 1986; Perkins, 1973; Pirenne, 1970) have said that observers do not notice the pictorial distortion afforded to a casual viewpoint because they are able to compensate for it. Knowing where the picture surface is, they make a mental allowance for the distortions that would be appropriate at their viewpoint of the moment. These compensation theories have been criticized severely on two counts: (1) their stated or implied reliance on an extra, picture-specific perceptual process of compensation and (2) evident failures of such compensation, most notably object rotation (Busey et al., 1990; Cutting, 1987, 1988; Halloran, 1989). The subtractive effect of frame rotation on object rotation is, in a sense, a compensation, though a limited one that does not contradict differential rotation. There is a rectifying principle, the comparison of object with frame, but not necessarily a separate rectification process. The frame, or picture surface, simply becomes the coordinate reference for orienting parts of the depiction, whenever the task suggests that this surface, rather than a frontal plane or the line of sight, be the orthogonal referent. Overall, rather than any purposeful compensation's taking place, the present work supports the general sense of Gogel's (1990) expectations that a "phenomenal geometry" involving depth relations would determine appearance in pictures as elsewhere, and that the perceptual processes are indifferent to the source (2-D or 3-D) of that geometrywith the caveat given here, however, that the multideterminate nature of perspective cues (Halloran, 1989) may require of people looking at pictures that they resolve conflicts of kinds not occurring in 3-D scenes.

Some successful trompe-l'oeil paintings (Gombrich, 1978; Pirenne, 1970) have scenes, and objects within scenes, of relatively little virtual depth. Such pictures will have minimal net object-to-frame rotation. It is unclear how this would make them "lifelike," as such pictures are said to be, especially since their objects still have a telltale constancy of aspect over viewing positions. It may be, however, as both Gombrich and Pirenne have observed in different ways, that we only or mostly notice constant aspect when we also notice that things follow us- 
that is, rotate-which they may do minimally in trompel'oeil. (For some trompe-l'oeil paintings, a relatively fixed viewpoint is contrived; see Pirenne, 1970.)

As may be inferred from the case of trompe-l'oeil, the fact of constant aspect does not explain why rotation is differential, since, presumably, aspect is equally constant for all depictions. However, it may not be quite as constant as supposed. The fragmentations of perspective indications of orientation, which occur in nonstandard arrays (Halloran, 1989), are clearly related to aspect, even though orientation and aspect are not the same thing: A perspective cue that specifies an orientation must also imply and specify a particular aspect-but it will not necessarily show that aspect, since the aspect may involve a face that is not included in the cue: the end of a box, for example, which might not be visible even though the perspective of its side is such that it should be. If aspect is not (analytically) constant, aspect may explain differential rotations (depth may even be discerned as aspect, or vice versa). At the same time, what constancy there is may explain depthless-object rotations, since constancy itself cannot depend on depth. Aspect could thus be a single, all-encompassing rotational factor. But much empirical and analytical work would be needed, to find out.

Strictly speaking, constant aspect is not a property of frames (except depicted ones), and they rotate also. Frame rotation would seem to be an instance of the phenomenal regression of slant toward a frontal plane that Thouless (1931) found for slanted plane forms and that is "a common but not inevitable occurrence" (Perkins, 1982) in experimental studies of slant.

It may be possible to account for phenomenal regression, and frame rotation, in terms of regular distortions in the perception of visual angles. Higashiyama (1992) has developed formulae for estimating the perceived size of visual angles when their actual size is known, based on findings that the angles tend to be seen as larger than they are, and that this is more true of vertical than of horizontal angles, and of smaller angles than of larger ones. This means that picture frames, for example, should appear at less of a slant from us than they really have, which is the way they do appear. The effect of visual-angle distortion on perspective geometry is complex, but regular dimensional enlargement is evidently the most important factor, enhanced by the fact that the farther vertical edge of a picture, being smaller, will be more enlarged perceptually than will be the nearer edge. The enlargement has the effect of moving the implicit vanishing point (the point at which parallels would appear to meet on the horizon) of the top and bottom edges of the frame (as seen from aside in projection) farther to the observer's side, and the greater enlargement of smaller angles increases the effect. The position of the vanishing point of parallels specifies the angle from the observer at which the parallels recede into depth. The farther leftward or rightward this point is, the more nearly frontal is the picture frame.

Interpolating from the left-hand plots in Figure 8 of Higashiyama (1992), the perceived visual angles of the inner border of the picture in the present Experiment 3 were estimated. The real visual angles were, for the near (vertical) edge, $11.3^{\circ}$; for the far edge, $8.9^{\circ}$; for the horizontal distance between them, $7.9^{\circ}$. From the plots, the predictions for the perceived visual angles would be, roughly, $22.8^{\circ}, 18.7^{\circ}$, and $14.8^{\circ}$, respectively. If it is assumed that viewing distance to the picture is correctly perceived, this enlarged size specifies a vanishing point, in virtual space, that is $52^{\circ}$ to the left of the line of sight, versus $30^{\circ}$ at the veridical frame size. This shallower convergence (more leftward) in turn specifies a picture that is slanted only $38^{\circ}$ from a frontal plane, instead of the actual $60^{\circ}$ slant. The $22^{\circ}$ rotation predicted by this calculation is substantially more than the frame rotation $\left(13.5^{\circ}\right)$ actually observed in Experiment 3, but approximately agrees with the frame rotations found in Experiments 2 and 4, for which the perspective-geometry values, and the prediction from the data of Higashiyama, would be similar.

It is not obvious, however, how or whether visual-angle distortion could account for differentials in object rotation. The projective geometry is just too complex, and the virtual space of a nonstandard view is actually multideterminate in certain respects that might be important to the needed analyses (Halloran, 1989). It seems clear, though, that not every individual distortion would support differential rotation. For example, a misorientation of the front edge of the side-viewed square figure in Experiments 1 and 2 (i.e., of its angle with the horizon), which is predictable from Higashiyama (1992), would specify exactly the same rotation for a horizontal as for an inclined square (assuming that the square continued to be perceived as having that edge horizontal); their front edges are identical in every respect of depiction and projection.

On the other hand, visual-angle enlargements (Higashiyama, 1992) might be the reason the arrows rotated more than the frame in Experiment 4; the arrows were smaller than the frame, and their rotation-inducing perspective distortions would have been correspondingly larger. Phenomenal enlargement meets the criteria suggested for a depth-free rotational factor, in Experiment 5: It is effective regardless of whether the object has virtual depth, it does not imply any relation of object with frame, and it is not, at least not obviously, implicated in producing rotational differentials.

Derȩgowski and Parker (1992) found that subjects misjudge the orientation of oblique contours that are presented to them in 2-D. They report this as evidence that such lines are immediately seen as being in 3-D. Whether this is so, distortions of visual angles (Higashiyama, 1992) might also cause these kinds of misjudgments. It is likely that the misorientations of oblique contours (Derȩgowski \& Parker, 1992), the frame rotations found here, and visualangle distortions (Higashiyama) are related phenomena.

It has been a dilemma in picture perception that devices for gaining precision by restricting exactly what is seen, such as monoculars, chinrests, and depictions extending 
beyond the field of view, also are known to produce an array-determined appraisal of the scene, supposedly because they reduce awareness of the picture surface (Pirenne, 1970). Since the array has now been shown to determine orientations, at least, in all picture perception, ordinary or reduced, there can be less basis for avoiding restrictedviewing techniques. Moreover, while the interrelations of constant aspect, virtual depth, scene stability, and differential rotation remain to be understood, they now seem unlikely to involve picture-surface awareness.

There seems little doubt that the frame is an effective cue to picture-surface orientation. It has proved to be a sufficient cue, even when surface texture, sheen, shadowing, and/or shade, to the extent that they were present, would have indicated wrongly (Halloran, 1989; and herein). Whether it remains the strongest cue, or even a useful one, under all conditions of frame shape and other surface gradients is unknown.

\section{REFERENCES}

Attneave, F. (1971). Multistability in perception. Scientific American, 225, 62-71.

Busey, T. A., Brady, N. P., \& Cutting, J. E. (1990). Compensation is unnecessary for the perception of faces in slanted pictures. Perception \& Psychophysics, 48, 1-11.

Cutting, J. E. (1987). Rigidity in cinema seen from the front row, side aisle. Journal of Experimental Psychology: Human Perception \& Performance, 13, 323-334.

CutTing, J. E. (1988). Affine distortions of pictorial space: Some predictions for Goldstein (1987) that La Gournerie (1859) might have made. Journal of Experimental Psychology: Human Perception \& Performance, 14, 305-311.

DerȩGowski, J. B., \& PARKer, D. M. (1992). Three-space inference from two-space stimulation. Perception \& Psychophysics, 51, 397-403.

Ellis, S. R., Smith, S., \& McGreevy, M. W. (1987). Distortions of perceived visual directions out of pictures. Perception \& Psychophysics, 42, 535-544.
Gogel, W. C. (1990). A theory of phenomenal geometry and its applications. Perception \& Psychophysics, 48, 105-123.

GolDSTEIN, E. B. (1979). Rotation of objects in pictures viewed at an angle: Evidence for different properties of two types of pictorial space. Journal of Experimental Psychology: Human Perception \& Performance, $5,78-87$.

GoLDSTEIN, E. B. (1987). Spatial layout, orientation relative to the observer, and perceived projection in pictures viewed at an angle. Joumal of Experimental Psychology: Human Perception \& Performance, 13, 256-266.

GolDSTEIN, E. B. (1988). Geometry or not geometry? Perceived orientation and spatial layout in pictures viewed at an angle. Journal of Experimental Psychology: Human Perception \& Peformance, 14, 312-314.

GoMBRICH, E. H. (1978). Meditations on a hobby horse and other essays on the theory of art (3rd ed.). London: Phaidon.

Halloran, T. O. (1989). Picture perception is array-specific: Viewing angle versus apparent orientation. Perception \& Psychophysics, 45, 467-482.

HigashiY AMA, A. (1992). Anisotropic perception of visual angle: Implications for the horizontal-vertical illusion, overconstancy of size, and the moon illusion. Perception \& Psychophysics, 51, 218-230.

KuBovy, M. (1986). The psychology of perspective and Renaissance art. Cambridge: Cambridge University Press.

Perkins, D. N. (1973). Compensating for distortion in viewing pictures obliquely. Perception \& Psychophysics, 14, 13-18.

Perkins, D. N. (1982). The perceiver as organizer and geometer. In J. Beck (Ed.), Organization and representation in perception (pp. 7393). Hillsdale, NJ: Erlbaum.

PIRENNe, M. H. (1970). Optics, painting and photography. Cambridge: Cambridge University Press.

RosinsKI, R. R., \& FARBER, J. M. (1980). Compensation for viewing point in the perception of pictured space. In M. A. Hagen (Ed.), The perception of pictures: Vol. 1. Alberti's window (pp. 137-176). New York: Academic Press.

Thouless, R. H. (1931). Phenomenal regression to the real object. British Journal of Psychology, 21, 339-359.

(Manuscript received March 28, 1990; revision accepted for publication March 4, 1993.) 